\title{
COMBATE DE MALEZAS, EN MONOCULTIVO DE MANÍ FORRAJERO (Arachis pintoi) ${ }^{1}$
}

\author{
Eugenio Araya ${ }^{2}$, Jorge Elizondo ${ }^{2}$, Carlos Jiménez ${ }^{3}$, Anaité Quan ${ }^{2}$
}

\begin{abstract}
RESUMEN
Combate de malezas, en monocultivo de maní forrajero (Arachis pintoi). Este trabajo se realizó con el objetivo de generar información sobre la eficacia de cuatro tratamientos químicos (herbicidas) en el control de malas hierbas, en comparación con la deshierba manual y un tratamiento testigo, todos los tratamientos tuvieron un manejo en rotación con 6,5 Unidades Animales (12 animales de $250 \mathrm{~kg}$ por cinco horas), con base en un monocultivo de maní forrajero de una área de $1.080 \mathrm{~m}^{2}$, con un descanso de 30 días. Para la evaluación de las diferentes variables se utilizó un diseño experimental de bloques completos al azar con seis tratamientos y tres repeticiones por tratamiento. Con el fin de determinar la cobertura del cultivo, tolerancia al ingrediente activo, grado de control del tratamiento y cuantificar la composicion botánica de la pastura, se llevaron a cabo cuatro evaluaciones; 0 , 30, 60 y 90 días de la aplicación de los tratamientos. Previamente a cada muestreo, se hizo un inventario de las malezas presentes en cada una de las parcelas de tratamiento. Con los tratamientos glifosato y limpieza manual se obtuvo la mayor cobertura de maní forrajero. El maní forrajero toleró adecuadamente todos los tratamientos, pero el glifosato y la limpieza manual, respectivamente dieron el mejor control de malezas. La producción de materia seca de las malezas y su porcentaje en la pastura disminuyó con el tiempo de muestreo. La mayor produccion y porcentaje de materia seca, se obtuvo con el tratamiento testigo.
\end{abstract}

\begin{abstract}
Fighting weeds in mono-cultures of forage peanut (Arachis pintoi). We evaluated four chemical treatments (herbicides) used to fight weeds, as compared to manual weed picking and using a witness treatment. All treatments had a rotational handling with 6.5 animal units (12 animals of $250 \mathrm{~kg}$ per five haurs). We used a proteid bank or monoculture of forage peanut, in an arca of 1080 sq.mt., with a 30 days break. In order to evaluate the many variables, we used an experimen-tal design of complete random blocks, with six treatments and tree repetitions per treatment. In order to determine the coverage of the culture, its tolerance to the active ingredient and the level of treatment control, we carried out four evaluations: $0,30,60$, and 90 days of treatment application. We found differences $(\mathrm{P}<0.01)$ between treatments related to the degree of coverage of the forage peanut plants. With Gliphosate and manual weeding, we obtained a dominant coverage of forage peanut, while with the witness treatment the degree of coverage was inferior. The production of dry matter from the weeds, as well as their percentage in the pasture, decreased in a significant way $(\mathrm{P}<0.01)$, through the sampling process. With the witness treatment we obtained the largest amount of dry matter in the weeds, and also the highest percentage of weeds in the pasture.
\end{abstract}

\section{INTRODUCCIÓN}

El maní forrajero pertenece a la familia Fabacea y tribu Aeschynomeneae de la subfamilia Papilionaceae (Grof, citado por Van Heurck, 1990). Es una especie promisoria, perenne, de crecimiento postrado estolonífero, originaria de la Amazonia Brasileña. Fue recolectada por G. C. Pinto en 1954, en el valle del río Jequitinhonha, en la costa atlántica del Brasil (Argel, 1991).
El maní forrajero crece desde el nivel del mar hasta una altitud de $1.800 \mathrm{msnm}$. Se desarrolla bien en diferentes tipos de vegetación, desde los bosques abiertos hasta las sabanas y en regiones con más de $2.000 \mathrm{~mm}$ de precipitación anual, tanto en zonas de inundaciones como en zonas en las que el periodo de sequía no sobrepasa los cuatro meses. Se adapta a suelos ácidos con textura liviana a pesada (Grof, citado por Van Heurck, 1990). Presenta buen comportamiento en asociaciones, aumenta su

\footnotetext{
1 Presentado en la XLII Reunión Anual del PCCMCA en El Salvador, 1996. Parte de la tesis de grado en Zootecnia, Universidad de Costa Rica. Proyecto inscrito en la Vicerrectoóa de Investigación de la Universidad de Costa Rica. N 737-93-555

2 Estación Experimental de Ganado Lechero Alfredo Volio Mata, Facultad de Agronomía, Universidad de Costa Rica. San José, Costa Rica.

3 Director de la Escuela de Zootecnia, Facultad de Agronomía, Universidad de Costa Rica. San José, Costa Rica.
} 
contenido en la pastura aún en estado de baja presión de pastoreo; debido a su alta capacidad productora de semillas y al brote de hojas a partir de estolones enraizados, lo que evita que sean defoliadas completamente por los animales en pastoreo; además sus puntos de crecimiento están bien protegidos, lo que asegura la persistencia bajo pastoreo excesivo (Grof, citado por Van Heurck, 1990).

Bajo las condiciones edafoclimáticas de Guápiles, Roig (1989), indica rendimientos de materia seca de $976 \mathrm{~kg} / \mathrm{ha}$ por corte cada ocho semanas, y señala su capacidad de asociarse con gramíneas agresivas y persistir bajo pastoreo.

Accesiones de Arachis pintoi como CIAT 17434, han mostrado buena resistencia a plagas y enfermedades durante cinco años de observación en los Llanos Orientales de Colombia perteneciente al ecosistema de sabana (Grof, citado por Van Heurck, 1990). Igualmente en Guápiles, representativo del bosque húmedo tropical, los ataques de plagas no son de importancia económica (Roig, 1989). La calidad de esta leguminosa en términos de proteína cruda es de $17 \%$ y la DIVMS de 62\%, pudiendo variar esta última en función de la estación del año (28\% en la época seca y $60 \%$ en la época húmeda) (Lazcano y Thomas, citados por Van Heurck, 1990).

Hurtado (1988), indicó niveles de DIVMS y PC de $51 \%$ y $10,8 \%$ respectivamente, para el forraje ofrecido en la asociación de Estrella Africana (Cynodon nlemfluensis) con Arachis pintoi. Estas características hacen del Arachis una buena fuente de proteína. En un ensayo realizado en Guápiles (Roig, 1989) encontró para el A. pintoi CIAT 17.434 un contenido de proteína de $25,4 \%$ y una DIVMS de 58,5\%. Por otro lado en los LLanos de Colombia Grof (citado por Van Heruck, 1990) informó de $14,1 \%$ de proteína cruda y contenidos adecuados de minerales. Esta especie es altamente tolerable a la defoliación ejercida con altas cargas animales. Así con una carga de 2,3 U.A/ha (U.A $=420 \mathrm{~kg}$ ) se han obtenido ganancias de peso de 521 gramos/animal/día y de 627 kg/ha/año (Giraldo, 1991).

En las pruebas en pastoreo A. pintoi, soporta muy bien el pisoteo, mostrando como requisito de manejo en asociaciones con Brachiaria brizantha, el uso de cargas de 2,5 a 3,0 U.A./ha $(\mathrm{UA}=400 \mathrm{~kg})$. El potencial de producción animal mostrado por la especie sembrada en asocio con esta gramínea varía entre 650 y $750 \mathrm{~g} / \mathrm{animal} / \mathrm{día}$ de ganancia de peso y $9 \mathrm{~kg}$ de leche/vaca/día (Argel, 1991).

Los estudios de la calidad de A. pintoi con Brachiaria dictyoneura, Brachiaria brizantha y Brachiaria ruziziensis indicaron que las asociaciones de Arachis con Brachiaria humidicola y Brachiaria dictyoneura ofrecen forraje de alta calidad a través del año, que se traduce en altas ganancias de peso y altas producciones de leche
(CIAT, 1987). Una de las caracteósticas más sobresalientes de este forraje es la producción de semilla sexual de reserva a través del año, lo que permite una excelente persistencia y una gran ventaja para su uso en zonas con época seca muy marcada, por la regeneración de la plantación por medio del desarrollo de la semilla sexual.

Debido a que el control de malezas se ha convertido en una práctica obligatoria necesaria, tanto en las etapas de establecimiento como de mantenimiento de los pastizales, el control químico constituye una alternativa muy usada, dado una serie de beneficios que se derivan de su uso, tales como facilidad de empleo, poca utilización de mano de obra, combate oportuno y eficaz y su efecto residual, ya que la eliminación de dichas malezas por métodos tradicionales son bastantes costosas y solo se logra parcialmente, debido a que la mayoría de ellas puede rebrotar, por lo que el uso apropiado de herbicidas resulta altamente beneficioso.

A pesar de que el maní forrajero (A. pintoi), ha sido ampliamente evaluado en Costa Rica, no existe suficiente información acerca del uso de herbicidas en este cultivo, por lo tanto, se ha utilizado documentación obtenida de evaluaciones hechas en maní comercial (Arachis hipogea).

Un control eficaz de malezas en Arachis pintoi, se obtuvo con la mezcla de metolaclor y gramurón al 5\% (v/v), sin embargo, esta mezcla causó un daño severo al maní forrajero establecido por semilla Argel y Valerio (1992). El control de malezas de hoja ancha y grarníneas, se han obtenido con herbicidas en posemergencia, con el uso de tres herbicidas selectivos a las primeras, a saber, 2,4-DB (Butoxone) en dosis de 0,25 kg/ha, Fomesafén (Flex) a razón de $1 \mathrm{~kg} / \mathrm{ha}$ y Bentazón (Basagrán) en dosis de $1 \mathrm{~kg} / \mathrm{ha}$, mezclados con tres graminicidas, Fluazifop-butil (Fusilade) a $0,50 \mathrm{~kg} / \mathrm{ha}$, Fenoxaprop-etil (Furore) a $0,02 \mathrm{~kg} / \mathrm{ha}$ y Diclofop-metil (noxán) a $0,75 \mathrm{~kg} / \mathrm{ha}$. Todos los graminicidas trabajaron adecuadamente y el Fomesafén dentro de los selectivos a hoja ancha, se obtuvo el menor número de malezas Muñoz (1989). Por su parte, Chavarría (1990), utilizó la dosis de $0,75 \mathrm{~kg} / \mathrm{ha}$ de Fluazifop-butil $+0,50 \mathrm{~kg} / \mathrm{ha}$ de Bentazón y obtuvo buen control en la posemergencia.

El presente trabajo consistió en la evaluación de cuatro tratamientos químicos y un tratamiento mecánico para el control de malezas en maní forrajero (A. pintoi).

\section{MATERIALES Y MÉTODOS}

\section{Localización del experimento}

El presente trabajo de investigación se llevó a cabo en la Estación Experimental de Ganado Lechero Alfredo Volio Mata situada en el distrito de San Rafael, cantón de 
la Unión de 1 provincia de Cartago, a una altitud de $1.546 \mathrm{msnm}$. El área que se utilizó, fue de 1.080 metros cuadrados y se sembró en el mes de junio de 1992. Esta plantación fue con Arachis pintoi invadida fundamentalmente por malezas de hoja ancha.

El experimento se inició en el mes de junio de 1993 y se extendió hasta el mes de octubre del mismo año. Este periodo comprendió solamente condiciones de invierno.

Muestreo: Cada parcela de tratamiento tuvo tres metros de largo por tres metros de ancho. Esto permitió dividirlas en 36 subparcelas de muestreo de 50 X $50 \mathrm{~cm}$.

Se llevó a cabo un muestreo de la vegetación total presente a ras del suelo, en tres de las 36 subparcelas, tomadas al azar, con el fin de cuantificar los diferentes componentes botánicos dela pastura, a saber: maní forrajero, maleza de hoja ancha, gramínea y ciperácea.

Después de realizado el muestreo, se incluyó un grupo de 12 novillos, con un peso promedio de $250 \mathrm{~kg}$ (6,5 unidades animales), para que pastorearan durante cinco horas en el área de experimentación. Estos animales formaron parte de todos los tratamientos. Una vez concluido el tiempo de pastoreo, se llevó a cabo una uni formización de las malezas. Esta uniformización se hizo con el fin de cortar las malezas de porte alto, y para estimular el crecimiento de material vegetativo, con el fin de que los herbicidas tuvieran mayor efecto sobre las malezas.

Después de la uniforrnización, se llevó a cabo la aplicación de los tratamientos. Transcurridos 30 días después de esta primera aplicación de los tratamientos, se llevó a cabo un muestreo de la vegetación. Además, se volvieron a introducir los animales y luego se llevó a cabo una segunda aplicación de los tratamientos. Cuando transcurrieron 60 días después de la primera aplicación de los tratamientos, se realizó un muestreo de la vegetación. Las 6,5 HUA se volvieron a incluir en el área de experimentación.

Noventa días después de la primera aplicación de los tratamientos, se llevó a cabo el último muestreo. Previamente a cada muestreo, se hizo un inventario delas malezas presentes en cada una de las parcelas de tratamiento (Cuadros 5, 6 y 7).

\section{Tratamientos utilizados:}

1- Glifosato (Round-up), aplicado con mechero a $40 \%$ de concentración sobre las malezas sin recortar (GLIF).
2- Mezcla de Bentazón (Basagrán) + Fluazifop-butil (Fusilade) a dosis de 1,00 y $0,50 \mathrm{~kg}$ i.a./ha respectivamente (BAS+FFB).

3- Mezcla de ácido 2,4-Dicloro fenoxibutírico $(2,4-$ DB) + Fluazifop-butil (Fusilade) a dosis de 0,25 y $0,50 \mathrm{~kg}$ i.a./ha respectivamente $(2,4-\mathrm{DB}+\mathrm{FFB})$.

4- Mezcla de Paraquat + Diurón (Gramurón) a dosis de 1,69 litros de producto comercial por hectárea y a una concentración de $0,5625 \%$ (90cc por bomba de 16 litros) (PAR+DIU).

5- Limpieza manual.

6- Testigo (solamente el ingreso de los animales)

Los tratamientos fueron aplicados en dos ocasiones: a los 0 y 60 días después de la siembra.

\section{Diseño experimental}

El diseño experimental utilizado fue el de bloques completos al azar con seis tratamientos y tres repeticiones por tratamiento. Cada parcela tuvo una área de nueve $\mathrm{m} 2$. La significancia entre promedios se evaluó por medio de la prueba de Duncan paralas variables de respuesta que resultaron significativas $(\mathrm{P}<0,05)$. Los datos obtenidos en el experimento, se analizaron utilizando el programa estadístico ZAS.

\section{Variables evaluadas}

a. de cobertura o abundancia del A. pintoi por medio del muestreo visual. Para esta variable se utilizó la escala para evaluación visual (Soto, 1992) Cuadro 1.

b. grado de tolerancia del A. pintoi a cada tratamiento. Para esta variable se utilizó laescala para evaluaron visual (Soto, 1992) Cuadro 2.

c. grado de control que tuvo cada tratamiento sobre las malezas. Para esta variable se utilizó la escala para evaluación visual (Soto, 1992) Cuadro 3. Peso seco de maní, maleza de hoja ancha, hoja angosta y ciperáceas en $0,75 \mathrm{~m}^{2}$ ( 3 submuestras de 0,25 $\mathrm{m}^{2}$ ) a los $0,30,60$ y 90 días después de la primera aplicación de herbicidas.

\section{RESULTADOS Y DISCUSIÓN}

\section{Grado de cobertura o abundancia del maní forrajero}

Se presentaron diferencias $(\mathrm{P}<0,01)$, entre tratamientos con respecto al grado de cobertura del maní forrajero. Con los tratamientos 1 y 5 (Glifosato y limpie- 
Cuadro 1. Escala para valorar la cobertura del cultivo forrajero.

\begin{tabular}{clc}
\hline Valor & Cobertura $(\%)$ & Abundancia \\
\hline 1 & Ocasional & $<1$ \\
2 & Pocas & $1-5$ \\
3 & Común & $6-30$ \\
4 & Abundante & $31-66$ \\
5 & Dominante & $67-100$ \\
\hline
\end{tabular}

(Soto y Agüero, 1992).

Cuadro 2. Escala para valorar la tolerancia del cultivo forrajero, al ingrediente activo o tratamiento.

\begin{tabular}{cll}
\hline Valor & Síntoma & \multicolumn{1}{c}{ Daño } \\
\hline 1 & Muy severo & Reducción en población $>90 \%$ \\
2 & Severo & Reducción en población 70-90\% \\
3 & Moderado & Reducción en población 30-70\% \\
4 & Ligero & Quema hojas y enanismo \\
5 & Ninguno & Nada \\
\hline
\end{tabular}

(Soto y Agüero, 1992)

Cuadro 3. Escala para valorar el grado de control del herbicida o tratamiento, sobre las malezas.

\begin{tabular}{cll}
\hline Valor & Combate $(\%)$ & Control \\
\hline 1 & Pobre & $<=10$ \\
2 & Ligero & $11-29$ \\
3 & Moderado & $30-69$ \\
4 & Bueno & $70-89$ \\
5 & Excelente & $>=90$ \\
\hline
\end{tabular}

(Soto y Agüero, 1992).

za manual respectivamente), se obtuvo una cobertura de maní forrajero dominante, mientras que con el tratamiento testigo, el grado de cobertura del maní forrajero fue el más bajo (Figura 1). Esto se debe a que tanto Glifosato y limpieza manual, por la manera en que fueron aplicados, afectaron principalmente la población de malezas. El maní forrajero, por su capacidad regeneradora y debido a un cambio en su forma de crecimiento (al existir menor competencia por luz, espacio, agua y nutrimentos, se elongó menos y creció de una forma más postrada), aumentó su cobertura en el terreno.

\section{Tolerancia del maní forrajero}

Los valores para la tolerancia del maní forrajero corresponden a tres de los cuatro muestreos realizados; los que se llevaron a cabo 30, 60 y 90 días después de la primera aplicación de los tratamientos. No se encontraron diferencias $(\mathrm{P}>0,05)$ para la tolerancia del maní forrajero a cada tratamiento. El maní forrajero toleró adecuadamente todos los tratamientos (Figura 1). Estos resultados difieren de los obtenidos por Argel y Valerio (1992), quienes utilizando una mezcla de metolaclor y gramurón a15\% (v/v), obtuvieron altos índices de control de malezas y severos daños a las plantas de Arachis pintoi, particularmente al establecimiento con semilla botánica. Sin embargo, hay que considerar que la evaluación de esta variable se llevó a cabo 30 días después de la aplicación de los tratamientos, tiempo suficiente para que las plantas se hubiesen recuperado en caso de que algún tratamiento les hubiera causado daño.

\section{Combate de malezas}

En el combate de malezas, se encontraron diferencias $(\mathrm{P}<0,01)$ entre tratamientos. Se observa en el Cuadro 4, como con los tratamientos uno y cinco (Glifosato y limpieza manual respectivamente) se obtuvo el mejor control de malezas; en el caso de que Glifosato no solo eliminó la parte aérea sino también las partes subterráneas de la planta (Ashton y Crafts, 1981). Con los tratamientos dos y seis (mezcla de Basagrán + Fluazifop-butil y tratamiento testigo respectivamente), se obtuvo el control de malezas más pobre.

\section{Malezas contaminantes}

En los Cuadros 5, 6, 7 y 8, se encuentran clasificadas las principales malezas contaminantes presentes al inicio del experimento y durante la realización del mismo.

\section{Materia seca del maní forrajero}

Para la materia seca del maní forrajero no se encontraron diferencias significativas $(\mathrm{P}>0,05)$ entre muestreos, lo cual indica que la acción de los tratamientos no afectó significativamente la producción del cultivo forrajero (Figura 2).

\section{Cantidad de materia seca de las malezas}

Hubo diferencias $(\mathrm{P}<0,01)$ para la cantidad de materia seca entre tratamientos. Con el Glifosato se obtuvo la cantidad más baja de malezas, sin embargo, este valor no difirió estadísticamente al obtenido con los tratamientos 2, 3, 4 y 5 (bentazón + fluazifop-butil, ácido 2,4-dicloro fenoxibutírico, paraquat + diurón y 


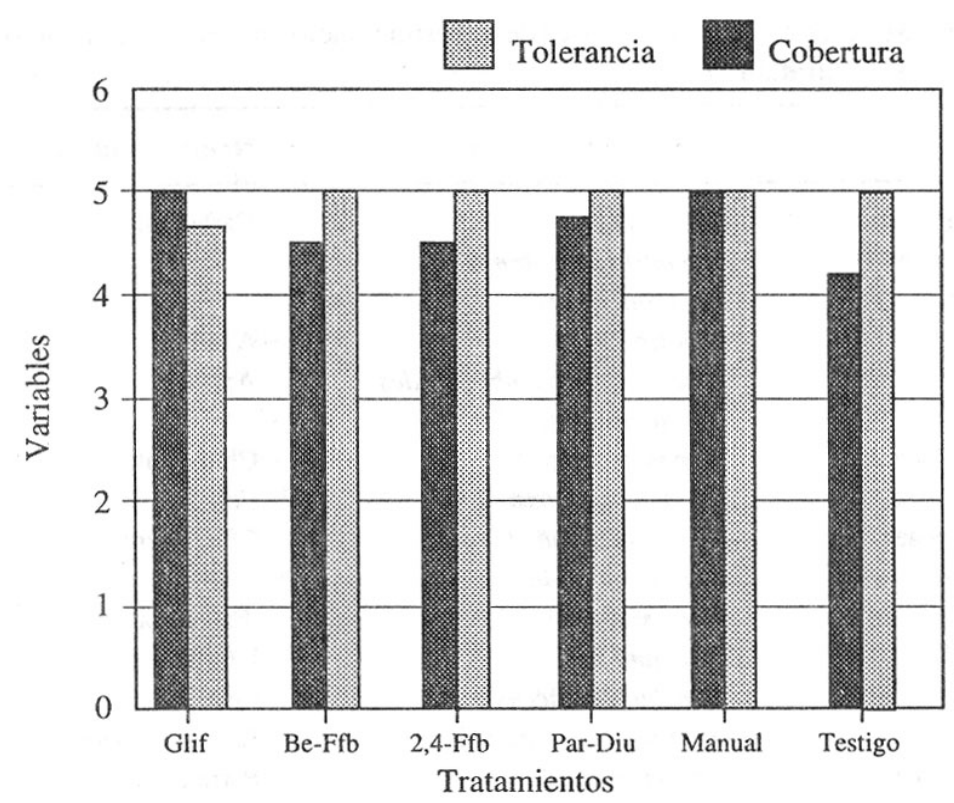

Fig. 1. Efecto de los diferentes tratamientos sobre las variables visuales evaluadas en Maní forrajero.

Cuadro 4. Efecto de la aplicación de los tratamientos sobre las diferentes variables evaluadas.

\begin{tabular}{lcccccc}
\hline & \multicolumn{6}{c}{ Tratamiento } \\
\cline { 2 - 7 } Variable & $\begin{array}{c}\mathbf{1} \\
\text { GLIF }\end{array}$ & $\begin{array}{c}\mathbf{2} \\
\text { BEN+FFB }\end{array}$ & $\begin{array}{c}\mathbf{3} \\
\mathbf{2 , 4 + F F B}\end{array}$ & $\begin{array}{c}\mathbf{4} \\
\text { PAR+DIU }\end{array}$ & $\begin{array}{c}\mathbf{5} \\
\text { Manual }\end{array}$ & $\begin{array}{c}\mathbf{6} \\
\text { Testigo F }\end{array}$ \\
\hline Cobertura & $5,00 \mathrm{a}$ & $4,50 \mathrm{c}$ & $4,50 \mathrm{c}$ & $4,75 \mathrm{~b}$ & $5,00 \mathrm{a}$ & $4,17 \mathrm{~d} *$ \\
Tolerancia & 4,67 & 5,00 & 5,00 & 5,00 & 5,00 & $5,00 \mathrm{~ns}$ \\
Control & $5,00 \mathrm{a}$ & $2,78 \mathrm{~d}$ & $3,33 \mathrm{c}$ & $4,00 \mathrm{~b}$ & $4,89 \mathrm{a}$ & $2,73 \mathrm{~d} *$ \\
\hline
\end{tabular}

ns : No significativo $(\mathrm{P}>0,05)$

* : Significativo $(\mathrm{P}<0,01)$

a, b, c, d : Medias con diferente letra en una misma hilera difieren estadísticamente.

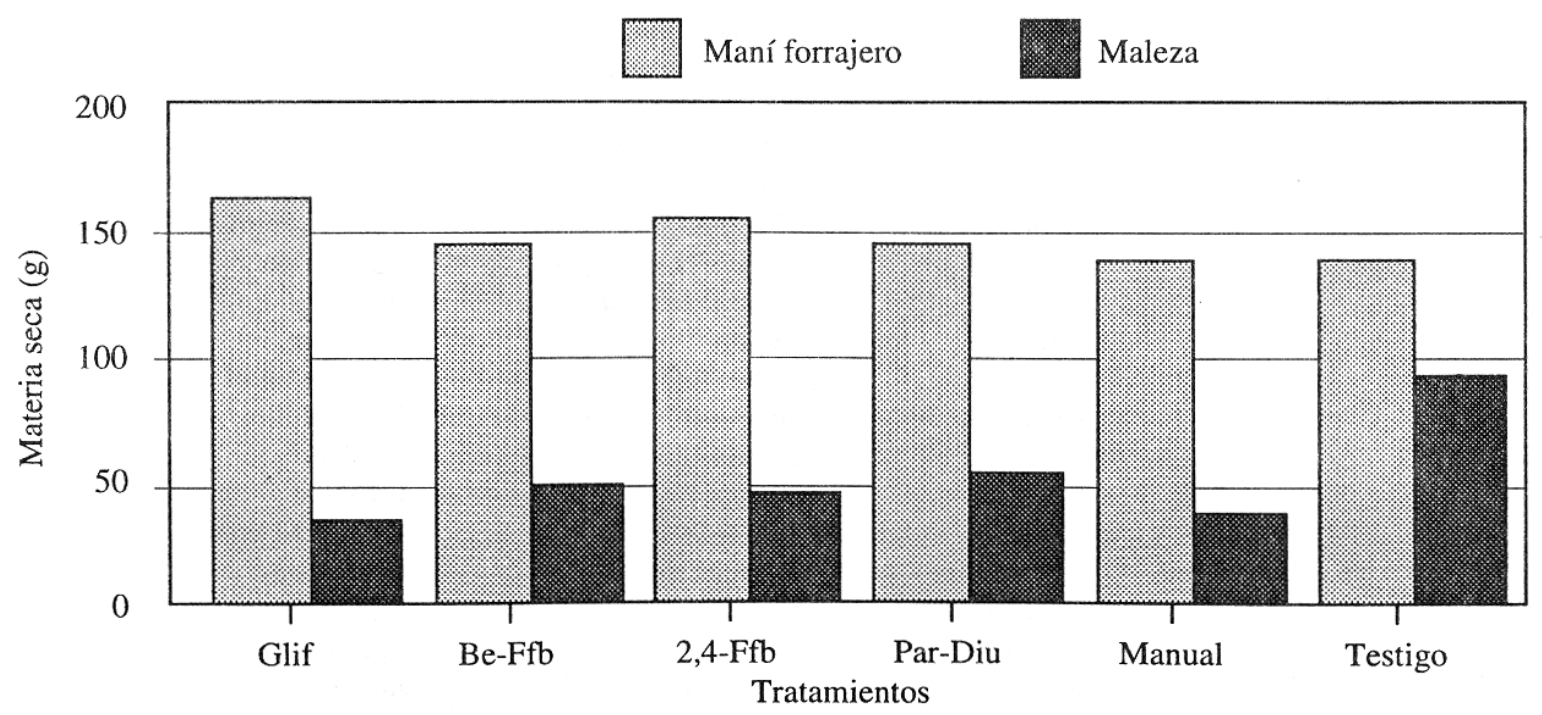

Fig. 2. Efecto de los tratamientos sobre la materia seca del maní forrajero y las malezas. 
Cuadro 5. Malezas presentes en el área de experimentación antes de la aplicación de los tratamientos.

\begin{tabular}{lll}
\hline Familia & Nombre Científico & Nombre Vulgar \\
\hline Fabaceae & Acacia spp & Carboncillo \\
Cariophylacea & Arenaría lanuginosa & \\
Cyperaceae & Cyperus tenuis & \\
Asteraceae & Coniza spp & Agento \\
Chenopodiaceae & Chenopodium ambrosioides & Apazote \\
Asteraceae & Gnaphalium spp & \\
Convolvulaceae & Hipomoea spp & Churristate \\
Phytolaccacea & Phytolacca icossandra & Frutilla \\
Polygonaceae & Polygonum punctatum & Chile perro \\
Malvaceae & Sida lichiopolia & \\
Malvaceae & Sida spp & Escobilla \\
Solanaceae & Solanum spp & Uña de Gato \\
Asteraceae & Sonchus oleraceus & Cerrajilla \\
Rubiaceae & Spermacoce assurgens & Juana la blanca \\
Cariophyllacea & Spergula arriensis & Perra parida \\
Fabacea & Teramnus uncinatus & Juanita \\
Verbenaceae & Verbena littoralis & Cola de Alacrán \\
Solanaceae & Verbesina turbacensis & \\
\hline
\end{tabular}

Cuadro 6. Malezas presentes en las parcelas 30 días después de la primera aplicación de los tratamientos.

\begin{tabular}{|c|c|c|c|}
\hline Parcela & Familia & Nombre Científico & Nombre Vulgar \\
\hline \multirow[t]{4}{*}{1} & Carophylacea & Arenaría lanuginosa & \\
\hline & Polygonaceae & Polygonum punctatum & Chile de Perro \\
\hline & Malvaceae & Sida spp & Escobilla \\
\hline & Fabaceae & Teramnus uncinatus & Juanita \\
\hline \multirow[t]{3}{*}{2} & Polygonaceae & Polygonum punctatum & Chile de Perro \\
\hline & Malvaceae & Sida $\mathrm{spp}$ & Escobilla \\
\hline & Verbenaceae & Verbena littoralis & Cola de Alacrán \\
\hline \multirow[t]{5}{*}{3} & Convolvulaceae & Hipomoea spp & Churristate \\
\hline & Polygonaceae & Polygonum punctatum & Chile de Perro \\
\hline & Fabaceae & Teramnus uncinatus & Juanita \\
\hline & Malvaceae & Sida spp & Escobilla \\
\hline & Verbenaceae & Verbena littoralis & Cola de Alacrán \\
\hline \multirow[t]{6}{*}{4} & Convolvulaceae & Hipomoea spp & Churristate \\
\hline & Polygonaceae & Polygonum punctatum & Chile de Perro \\
\hline & Rubiaceae & Spermacoce assurgens & Juana la blanca \\
\hline & Fabaceae & Teramnus uncinatus & Juanita \\
\hline & Malvaceae & Sida $\mathrm{spp}$ & Escobilla \\
\hline & Verbenaceae & Verbena littoralis & Cola de Alacrán \\
\hline \multirow[t]{4}{*}{5} & Polygonaceae & Polygonum punctatum & Chile de Perro \\
\hline & Fabaceae & Teramnus uncinatus & Juanita \\
\hline & Malvaceae & Sida spp & Escobilla \\
\hline & Verbenaceae & Verbena littoralis & Cola de Alacrán \\
\hline \multirow[t]{6}{*}{6} & Convolvulaceae & Hipomoea spp & Churristate \\
\hline & Phytolaccaceae & Phytolacca icossandra & Frutilla \\
\hline & Polygonaceae & Polygonum punctatum & Chile de Perro \\
\hline & Fabaceae & Teramnus uncinatus & Juanita \\
\hline & Malvaceae & Sida spp & Escobilla \\
\hline & Verbenaceae & Verbena littoralis & Cola de Alacrán \\
\hline
\end{tabular}


Cuadro 7. Malezas presentes en las parcelas 60 días después de la primera aplicación de los tratamientos.

\begin{tabular}{clll}
\hline Parcela & Familia & Nombre Científico & Nombre Vulgar \\
\hline 1 & Malvaceae & Sida spp & Escobilla \\
& Verbenaceae & Verbena littoralis & Cola de Alacrán \\
2 & Polygonaceae & Polygonum punctatum & Chile de Perro \\
& Malvaceae & Sida spp & Escobilla \\
& Fabaceae & Teramnus uncinatus & Juanita \\
& Phytolaccaceae & Phytolacca icossandra & Frutilla \\
& Polygonaceae & Polygonum punctatum & Chile de Perro \\
& Fabaceae & Teramnus uncinatus & Juanita \\
& Malvaceae & Sida spp & Escobilla \\
& Polygonaceae & Polygonum punctatum & Chile de Perro \\
& Rubiaceae & Spermacoce assurgens & Juana la blanca \\
& Fabaceae & Teramnus uncinatus & Juanita \\
& Malvaceae & Sida spp & Escobilla \\
& Polygonaceae & Polygonum punctatum & Chile de Perro \\
& Phytolaccaceae & Phytolacca icossandra & Frutilla \\
& Polygonaceae & Polygonum punctatum & Chile de Perro \\
& Fabaceae & Teramnus uncinatus & Juanita \\
& Malvaceae & Sida spp & Escobilla \\
& Rubiaceae & Spermacoce assurgens & Juana la blanca \\
& Verbenaceae & Verbena littoralis & Cola de Alacrán \\
\hline & & & \\
& & & \\
& & &
\end{tabular}

Cuadro 8. Malezas presentes en las parcelas 90 días después de la primera aplicación de los tratamientos.

\begin{tabular}{clll}
\hline Parcela & Familia & Nombre Científico & Nombre Vulgar \\
\hline 1 & Cyperaceae & Cyperus tenuis & \\
& Malvaceae & Sida spp & Escobilla \\
& Polygonaceae & Polygonum punctatum & Chile de Perro \\
& Polygonaceae & Polygonum punctatum & Chile de Perro \\
& Malvaceae & Sida spp & Escobilla \\
& Rubiaceae & Spermacoce assurgens & Juana la blanca \\
& Verbenaceae & Verbena littoralis & Cola de Alacrán \\
& Polygonaceae & Polygonum punctatum & Chile de Perro \\
& Malvaceae & Sida spp & Escobilla \\
& Polygonaceae & Polygonum punctatum & Chile de Perro \\
& Rubiaceae & Spermacoce assurgens & Juana la blanca \\
& Fabaceae & Teramnus uncinatus & Juanita \\
& Verbenaceae & Verbena littoralis & Cola de alacrán \\
& Polygonaceae & Polygonum punctatum & Chile de Perro \\
& Malvaceae & Sida spp & Escobilla \\
& Convolvulaceae & Hipomoea spp & Churristate \\
& Phytolaccaceae & Phytolacca icossandra & Frutilla \\
& Polygonaceae & Polygonum punctatum & Chile de Perro \\
& Fabaceae & Teramnus uncinatus & Juanita \\
& Malvaceae & Sida spp & Escobilla \\
& Rubiaceae & Spermacoce assurgens & Juana la blanca \\
& Verbenaceae & Verbena littoralis & Cola de Alacrán \\
\hline & & &
\end{tabular}


limpieza manual respectivamente) (Figura 2). Con el tratamiento testigo, se obtuvo la mayor cantidad de materia seca de las malezas lo cuál era de esperar, ya que los animales muestran mayor preferencia por el maní forrajero y muy probablemente no consuman una serie de malezas presentes, además, con el paso del tiempo es de esperar que la cantidad de malezas aumente al ser más agresiva que el cultivo forrajero.

En el primer muestreo, se obtuvo la mayor cantidad de materia seca de malezas y ésta fue disminuyendo a través de los muestreos. En el tercer y cuarto muestreo se obtuvo la cantidad más baja, quedando bien establecido el efecto negativo de los tratamientos sobre la población de malezas (Figura 3).

\section{Porcentaje del maní forrajero en la pastura}

Se presentaron variaciones $(\mathrm{P}<0,01)$ entre muestreos. En el primer muestreo se obtuvo el menor porcentaje de maní forrajero, mientras que en el tercer y cuarto muestreo el porcentaje obtenido fue el mayor (Figura 4). Esto se atribuye al efecto detrimental de los tratamientos sobre las malezas, ya que después de la aplicación de éstos, la cantidad de materia seca del maní forrajero se mantiene casi constante, mientras que la cantidad de materia seca de las malezas disminuyó considerablemente, reduciéndose así su relación porcentual en la pastura.

Se presentaron variaciones significativas $(\mathrm{P}<0,01)$ entre tratamientos, para el porcentaje de maní forrajero. El tratamiento 1 (Glifosato) presentó el mayor por- centaje, aunque éste no difirió estadísticamente de los tratamientos 3, 4 y 5 (ácido 2,4-Dicloro fenoxibutírico, paraquat + diurón, limpieza manual respectivamente), (Figura 5), con el tratamiento testigo, se obtuvo el menor porcentaje de maní forrajero, debido a que con este mismo tratamiento se obtuvo la mayor cantidad de materia seca de malezas.

\section{Porcentaje de malezas en la pastura}

Se observaron diferencias $(\mathrm{P}<0,01)$ entre muestreos para el porcentaje de malezas, observándose que en el primer muestre o se obtuvo el mayor porcentaje de malezas, mientras que en el tercero y cuarto muestren el porcentaje de malezas obtenido fue el más bajo (Figura 4).

Se observaron diferencias $(\mathrm{P}<0,01)$ entre tratamientos para esta variable, observándose que con el tratamiento 1 (glifosato) se obtuvo el menor porcentaje de malezas, mientras que con el tratamiento seis (tratamiento testigo) se obtuvo el mayor porcentaje (Figura 5).

\section{Análisis económico}

En el Cuadro 9 se encuentra el análisis utilizado para determinar la efectividad de cada tratamiento en términos económicos. Puede observarse en dicho cuadro como el tratamiento 1 (glifosato) resultó ser el más efectivo en términos económicos, además de que con este tratamiento se obtuvo el mejor control de malezas. En tanto, el se obtuvo el mejor control de malezas. En

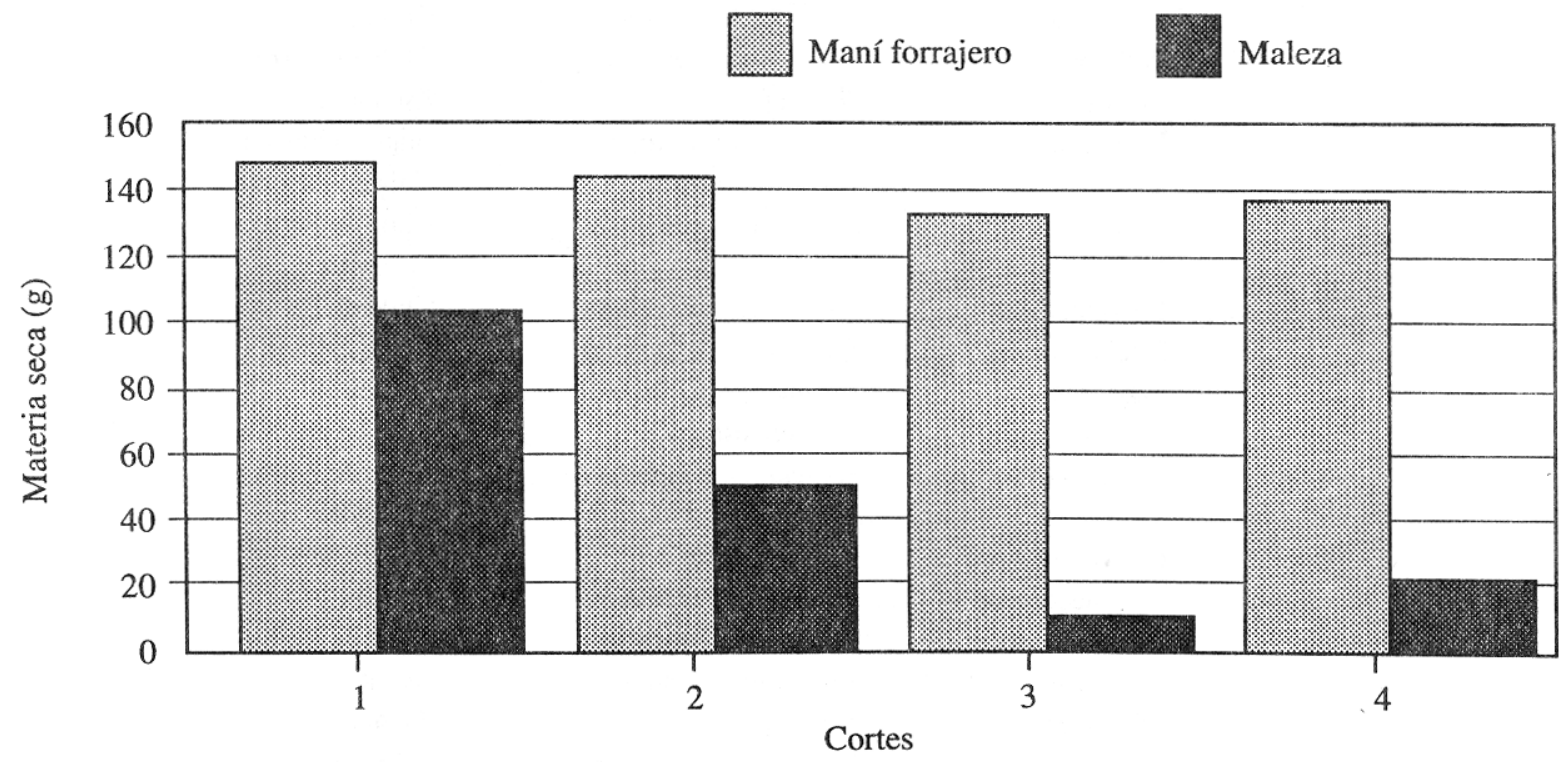

Fig. 3. Efecto de diferentes cortes, sobre la cantidad de materia seća de los diferentes componentes. 


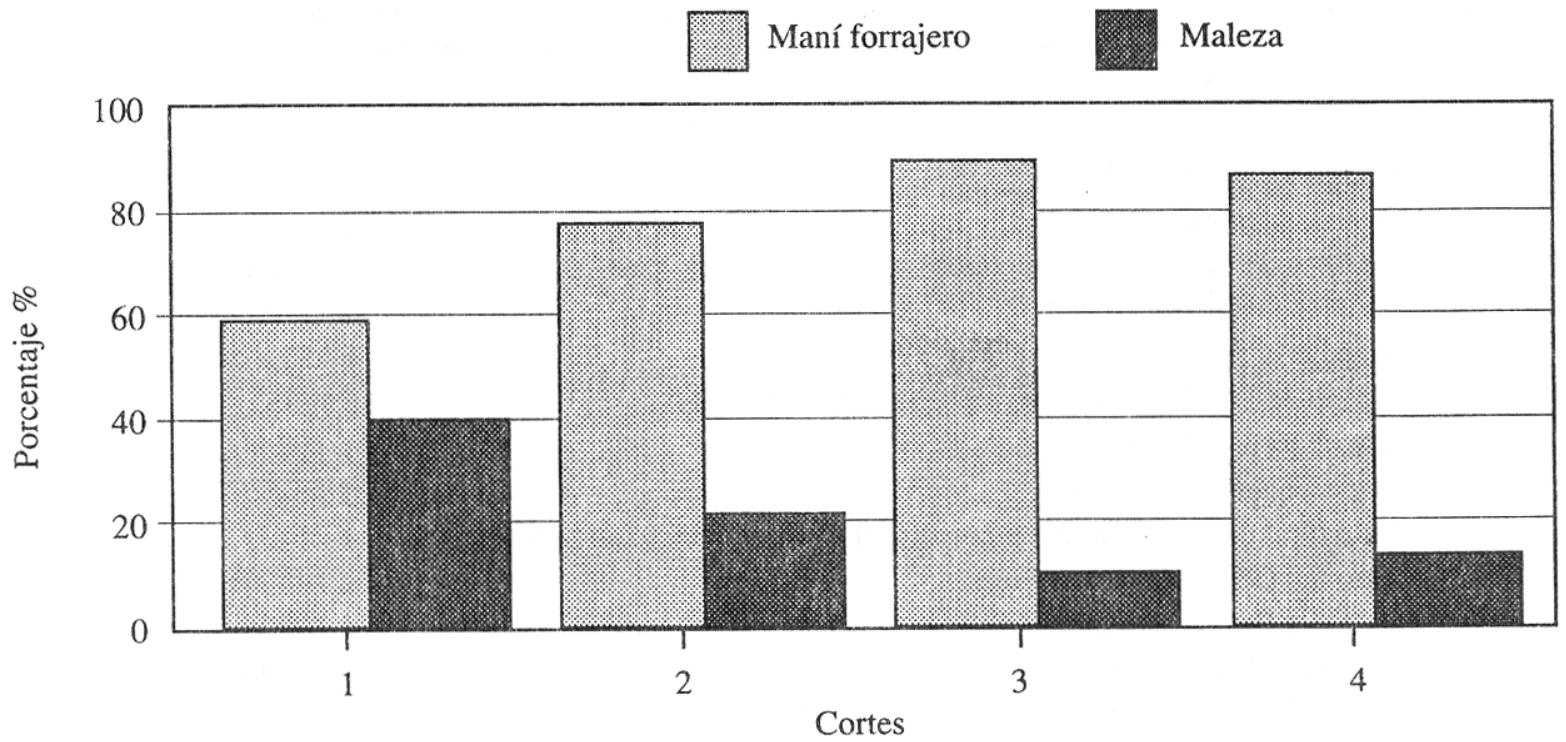

Fig. 4. Efecto de número de cortes sobre porcentajes de maní forrajero y maleza.

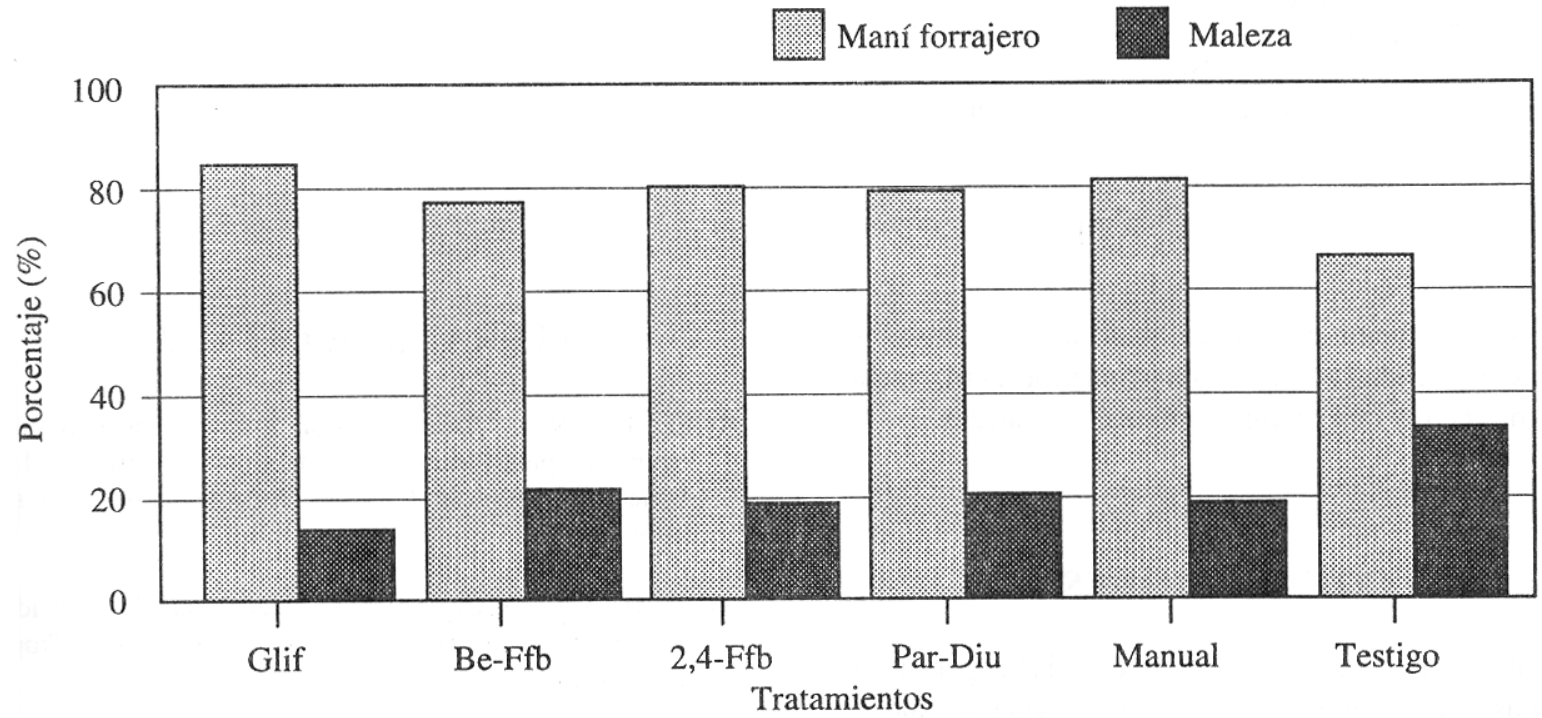

Fig. 5. Efecto de los tratamientos sobre el porcentaje de maní forrajero y maleza.

Cuadro 9. Costos (en colones) utilizados para determinar la efectividad de cada tratamiento en términos económicos.

\begin{tabular}{rrrrrr}
\hline Trat. & $\begin{array}{c}\text { Aplicación } \\
\text { de tractor }\end{array}$ & $\begin{array}{c}\text { Mano de } \\
\text { obra }\end{array}$ & $\begin{array}{c}\text { Costo del } \\
\text { producto }\end{array}$ & $\begin{array}{c}\text { Costo } \\
\text { total }\end{array}$ & $\begin{array}{c}\text { Grado de } \\
\text { control * }\end{array}$ \\
\hline 1 & 2331 & 217 & 5272 & 7820 & 5,00 \\
2 & 3567 & 217 & 17968 & 21752 & 2,78 \\
3 & 3567 & 217 & 14263 & 18047 & 3,33 \\
4 & 3567 & 217 & 5452 & 9236 & 4,00 \\
5 & - & 8000 & - & 8000 & 4,89 \\
6 & - & - & - & - & 2,73 \\
\hline
\end{tabular}

* Estos valores corresponden a las medias obtenidas por la prueba de Duncan $(\mathrm{P}<0,05)$, en una escala de 1 a 5 (ver Cuadro 4). 
Cuadro 10. Variables utilizados en el cálculo de los costos de aplicación (155 Colones / US \$1).

\begin{tabular}{ll}
\hline Tamaño del boom: & 3,0 metros. \\
Boquilla \#: & 8004 \\
Distancia entre boquillas: & $20 \mathrm{~cm}$. \\
Altura del boom: & $60 \mathrm{~cm}$. \\
Volumen de descarga por hectárea*: & $300 \mathrm{litros}$. \\
Velocidad de aplicación**: & $15 \mathrm{~km} / \mathrm{h}$. \\
Costo de la aplicación con tractor***: & $\phi 45 / \mathrm{galón}$. \\
Tiempo de lavado del equipo y preparación del caldo: & 1 hora. \\
Costo hora-hombre en jornal de 6 horas: & $\phi 158,90$. \\
Cargas sociales(36,66\%): & $\phi 58,27$. \\
Costo total de hora laboral en lavado del equipo y preparación del caldo: & $\phi 217$. \\
Costo del Glifosato (Round-up): & $\phi 1687 /$ litro. \\
Costo del Basagrán (bentazón): & $\notin 3030 /$ itro. \\
Costo del Fluazifop-butil (Fusilade): & $\phi 3950 /$ litro. \\
Costo del ácido 2,4-dicloro fenoxibutírico (2,4-DB): & $\varnothing 2190 /$ litro. \\
Costo del paraquat + diurón (Gramurón): & $\phi 1004 /$ itro. \\
Costo de la limpieza manual: & $\phi 8000 / \mathrm{ha}$.
\end{tabular}

Costos al lero de marzo de 1994.

* 7,81 Litros para el glifosato (tratamiento 1).

$* * 10 \mathrm{~km} / \mathrm{h}$ para el glifosato.

$* * * \notin 700 / \mathrm{km}$ para la aplicación con mechero.

tanto, el tratamiento 2 (mezcla de bentazón y fluazifopbutil) resultó ser el más caro y con un ligero control de malezas.

\section{CONCLUSIONES}

Bajo las condiciones en que se realizó el experimento y con base en los datos obtenidos en el presente trabajo, se concluye que:

1- El buen control de malezas incrementó la cobertura y biomasa del maní forrajero. El Glifosato en aplicación dirigida con mechero y limpieza manual favorecieron una mayor cobertura el maní y fueron los mas efectivos en controlar las malezas.

2- La mejor cobertura de maní forrajero se obtuvo con los tratamientos 1 y 5 (Glifosato y limpieza manual respectivamente), mientras que con el tratamiento testigo se obtuvo la cobertura más pobre.

3- El maní forrajero toleró todos los tratamientos aplicados en el experimento.

\section{LITERATURA CITADA}

ARGEL, P. 1991. Arachis pintoi: a new tropical pasture legume. Tropical Pasture Program. Centro Internacional de Agricultura Tropical (CIA T). Documento borrador sin editar.

ARGEL, P.; V ALERIO, A. 1992. Selectividad de herbicidas en el control de malezas en Arachis pintoi. Pasturas Tropicales. 14(2):23-26.

ASHTON, F. ; CRAFTS, A. 1981. Mode of action of herbicides. 2 da ed. Nueva York, Estados Unidos. John Willey and Sons. 525 p.

CIAT. 1987. Programa de pastos tropicales. Informe anual 1986. Cali, Colombia. 347 p.

CHAVARRíA, W. 1990. Evaluación agroeconómica de tratamientos químicos y físicos para el control de malezas en maní (Arachis hipogaea L). Tesis presentada para optar al título de Ingeniero Agrónomo con énfasis en Fitotecnia. Universidad de Costa Rica. San Pedro, San José. 75 p.

GIRALDO, L. 1991. Evaluación bajo pastoreo de la gramínea Brachiaria brizantha CIAT 6780 establecida sola o en asocio con Arachis pintoi CIAT 17434 manejadas bajo dos cargas animales, en el trópico húmedo de Costa Rica. Tesis Mg. Sc. CATIE. Turrialba, Costa Rica. 144 p. 
HURTADO, J. 1988. Introducción de leguminosas y manejo del pastoreo. Tesis Mg. Sc. CATIE. Turrialba, Costa Rica. $107 \mathrm{p}$.

MUÑOZ, L. 1989. Evaluación de mezclas de herbicidas preemergentes y posemergentes en maní (Arachis hipogaea L.) en la Estación Experimental Fabio Baudrit M. Tesis presentada para optar al título de Ingeniero Agrónomo con énfasis en Fitotecnia. Universidad de Costa Rica. San Pedro, San José. 85 p.

ROIG, C. 1989. Evaluación preliminar de 200 accesiones de leguminosas forrajeras tropicales en un ecosistema de bosque tropical lluvioso (Guápiles, Costa Rica). Tesis Mg. Se. CA TIE. Turrialba, Costa Rica. 179 p.
SOTO, A. 1992. Escalas para valorar la cobertura del cultivo forrajero, su tolerancia al ingrediente activo y el grado de control del herbicida. Comunicación personal.

SOTO, A.; AGÜERO, R. 1992. Combate químico de malezas en el cultivo de arroz. San José, Costa Rica. Editorial Universidad de Costa Rica. 81 p.

VAN HEURCK, L. 1990. Evaluación del pasto Estrella (Cynodon nlemfuensis) solo y asociado con las leguminosas forrajeras Arachis pintoi CIAT 17434 y Desmodium ovalifolium CIAT 350 en la producción de leche y sus componentes. Tesis Mg. Se. CATIE. Turrialba, Costa Rica. 111 p. 